\title{
Teaching and Learning Multiple Varieties of a Foreign Language for Sustainable Multilingual Education
}

\author{
Hao $X u^{1}(\mathbb{D}$ and Zhibin Shan $2,3, *$ (D) \\ 1 National Research Centre for Foreign Language Education, Beijing Foreign Studies University, \\ Beijing 100089, China; xuhaokent@bfsu.edu.cn \\ 2 Faculty of French and Francophone Studies, Beijing Foreign Studies University, Beijing 100089, China \\ 3 Laboratory STIH (Sens, Texte, Informatique, Histoire), Faculty of Arts and Humanities, Sorbonne University, \\ 75005 Paris, France \\ * Correspondence: shanzhibin@bfsu.edu.cn
}

check for updates

Citation: $\mathrm{Xu}, \mathrm{H}$.; Shan, Z. Teaching and Learning Multiple Varieties of a Foreign Language for Sustainable Multilingual Education. Sustainability 2021, 13, 8004. https://doi.org/ $10.3390 /$ su13148004

Academic Editors: Lawrence Zhang and Vincent T. Greenier

Received: 5 July 2021

Accepted: 16 July 2021

Published: 17 July 2021

Publisher's Note: MDPI stays neutral with regard to jurisdictional claims in published maps and institutional affiliations.

Copyright: (c) 2021 by the authors. Licensee MDPI, Basel, Switzerland. This article is an open access article distributed under the terms and conditions of the Creative Commons Attribution (CC BY) license (https:// creativecommons.org/licenses/by/ $4.0 /)$.

\begin{abstract}
In multilingual education for sustainable personal development, compared with that of multiple languages, the teaching and learning of multiple varieties of a language has been underexplored as a special and important form of multilingualism. In this article, we examine the linguistic, psychological, and social characteristics of multiple variety learning, as compared with multiple language learning. Linguistically, acquisition of language varieties is a process of assimilating variants from a new variety into an earlier variety, which serves as a prototype system. Such assimilation is a psychological project of form-meaning interface development, which may follow the patterns of structural multiplication, conceptual involution, conceptual evolution, or/and conceptual transfer. When multiple language varieties are actually used in social contexts, multilingual individuals' selected language practices may be supported by their combined linguistic resources from multiple varieties rather than depend on a single variety despite its dominance in a given situation. These characteristics carry pedagogical implications for sustainable multilingual education, particularly for the teaching and learning of foreign languages that have multiple varieties.
\end{abstract}

Keywords: multiple language varieties; multilingual literacies; multilingual education; sustainable development; multi-competences; form-meaning interface; the Dominant Language Constellations

\section{Introduction}

As a social phenomenon, multilingualism has attracted long-lasting attention from both sociolinguists and language teaching researchers and practitioners [1-3]. As valuable social capital, an individual's being multilingual, i.e., the state of individual multilingualism, reflects their knowledge and use of two or more languages, dialects of a single language, or varieties of a single dialect [4], thus providing them with linguistic advantages over those who are monolingual, particularly in multilingual contexts [5].

Beyond bringing linguistic advantages such as social capital, multilingualism contributes to individuals' sustainable personal development through multilingual literacies, which encompass linguistic multi-competences, world knowledge, and language awareness [6]. Although the three components of multilingual literacies are, in nature, different from literacies as real-life skills and practices, they all play essential roles in enabling literacy skills as a knowledge base or support individuals' social cognition. Numerous studies have revealed how individuals with multilingual literacies demonstrate better cognitive capabilities $[7,8]$ as well as greater potential for academic achievement $[9,10]$. These advantages can be gained and developed at different stages of life such as early childhood, adolescence, and adulthood, and can even be sustained throughout a lifetime [11]. Socioculturally, multilingual literacies enable an individual to own a linguistic repertoire, i.e., all the languages/dialects/varieties a person is capable of speaking, which can be activated by communication needs and mobilised in social interaction: when a new language, dialect, 
or variety is added into that repertoire as investment for economic or symbolic benefits, the individual's communicative capital will be enriched for socio-cultural empowerment [12].

Therefore, educational programmes worldwide that are oriented towards sustainable development have been implemented to foster learners' multilingual literacies. For instance, each European citizen is expected to become trilingual through formal education, mastering two European languages in addition to an official local language [12]; in the North American context, efforts have been made by schools and universities to help students, especially those whose L1 is not English, become multilingual by developing their academic English literacy for academic success [13]; in Greater China, educational policies pertaining to foreign languages are characterised by the predominance of English and a recent reinvigorated investment in teaching and learning languages other than English, an essential step to achieve multilingual literacies [14].

These endeavours, however, may have only partly addressed the need of multilingual literacies for sustainable development, as such literacies seem to be confined to those of multiple languages. In fact, multilingual literacies are needed also by individuals who learn and use multiple varieties of a language such as World Englishes and Global Frenches [15-17]. Such needs are underestimated, particularly in contexts where languages are taught and learnt as foreign languages, because a foreign language is often intuitively conceived as being a language of a "standard" variety [18,19]. Although multiple language varieties, as a particular type of multilingualism, conform to multiple languages in various ways, the teaching and learning of multiple varieties may bring about distinctive linguistic, psychological, and social issues that require more systematic inquiries. As to language learning, second or foreign language learning in particular, combined perspectives are highly preferable as they better reflect the interdisciplinary nature of such learning phenomena [20]. Previous studies on World Englishes and Global Frenches have successfully legitimised the spread and use of multiple varieties as sociolinguistic realities $[16,17,21]$, but they have rarely considered the possibilities and, more precisely, realities of multiple varieties being learnt and used by the same individual as a multilingual. Hence, researchers have been unable to address the issue of teaching and learning multiple language varieties as multilingual literacies for sustainable development.

To fill this gap in the literature, this article aims to delineate our theoretical considerations of multiple variety learning with tentative attempts to analyse the characteristics of multiple language varieties as taught and learnt to promote multilingual literacies. Specifically, this article will take combined perspectives that are compatible with second or foreign language learning to examine the teaching and learning of multiple varieties of a language as compared with that of multiple languages from linguistic, psychological, and social perspectives.

\section{Multiple Varieties Acquired as Systems of Variants}

Linguistically, the teaching and learning of multiple languages, or multilingual education in a wider sense, has been investigated focusing on two central issues. The first issue is concerned with the building of linguistic systems pertaining to languages. A linguistic system consists of both the principles and parameters, as is contended by the Universal Grammar (UG) [22]. The principles are what all human languages comply with; since the principles shared by all human languages do not need to be learnt, learning a new language should focus on acquiring parameters of that particular language, which are its unique features [22]. In other words, learning a new language resembles a process of setting new parameters of that language based on existing principles. Therefore, as is viewed in terms of the UG, it is important to examine how new parameters from the new language being acquired are set in relation to the existing system or systems pertaining to previously acquired language or languages. Are the new parameters incorporated into an existing system or systems, or do they construct an independent new system [22]? The second central issue is concerned with typological differences between languages, which indicates similarities between languages. Typological difference can be either linguistic 
and objective, when it is based on genetic relatedness between languages or comparison of particular linguistic features; or psychological and subjective, when generated from a learner's perception [23]. What, then, is the impact of typological difference between the acquired language(s) and the new language(s) being acquired on the difficulty of learning the new language(s) [24]? In this section, we will examine and reveal how the teaching and learning of multiple languages and that of multiple varieties of a language differ with regard to these two linguistic issues.

The UG contends that language acquisition or learning is a process of setting parameters of a specific language to the already existing principles of language in the brain that are genetically inherited and thus shared across all human languages [25]. When parameters of a specific language are gradually placed, a mental system pertaining to that language will emerge and develop [22]. Acquisition of a specific language is therefore an on-going project of moulding and enriching such a language-specific system until full competence is acquired, which means that the learner becomes a native speaker of that language [26]. When a learner learns a second or foreign language, parameters of the new language will be similarly processed and placed. This requires the brain to construct a new system for the new language. In the meantime, parameters of a previously acquired language will remain, and will not be replaced by parameters of the new language. The final result should be coexistence of two or more systems independent of each other, which pertain to both or all of the previously and newly acquired languages [27]. In other words, in the brains of bilingual or multilingual individuals, there exist more than one language-specific system. Even in situations of balanced bilingualism or multilingualism that involve two or more languages being simultaneously learnt rather than being learnt one after another, the coexistence of multiple language systems can also be the final result, although the process may be more complicated. Because the parameters of two or more languages need to be processed at the same time, occasional confusion on the learner's part may arise, and some parameters may be temporarily misplaced in another system, before they are finally re-placed in the correct, corresponding system [28].

Nonetheless, when it comes to the learning of multiple varieties of a language, the system-building process based on parameter setting may be fundamentally different. Highly probably, parameters of a new language variety may be assimilated into the existing system pertaining to a previously learnt variety. As parameters of different language varieties share substantial similarity or even sameness [29], contradiction between them and rejection of new parameters by the existing system are unlikely to occur frequently. Consequently, a new, independent system pertaining to the newly acquired language variety may not be easily developed.

This carries important implications for the teaching and learning of multiple varieties of a language. The learning of a new language variety is by nature assimilative, i.e., new parameters are added to an existing system without substituting its existing parameters. That means that no new grammatical or syntactic system will be constructed. Therefore, the teaching and learning of a new language variety should not be aimed at constructing a new rule-system. Rather, it should be intended for the cultivation of a new usage-system. When two or more varieties of a language are compared, grammatical or syntactic similarities often prevail over disparities [29]. Conversely, disparities in usage tend to outweigh similarities [30]. This requires the teaching and learning of a new language variety to emphasise differences in usage, rather than in grammar or syntax, as compared with a previously learnt variety.

Besides parameter setting, typological distance is also an important linguistic issue that warrants investigation so as to significantly contrast between multiple language learning and multiple variety learning. It has been established that typological distance between a previously acquired language and a language to be acquired afterwards is a major determinant of difficulty in acquiring the new language [31]. For the same reason, if an individual has acquired multiple languages that are characterised by diverse typological 
features, they should be able to acquire another additional language more easily, due to their richer typological reserve.

Typologically, different varieties of a language are intrinsically homogeneous, presumably featuring the closest typological distance, i.e., closest similarities, among themselves. This reduces the "typological barriers" to learning a new variety of a language when the learner has previously acquired another variety. Strictly speaking, differences between varieties of a language are not typological in nature, and contrastive analysis has been widely conducted to bring out variational changes from one variety to another, rather than typological differences as it were $[16,17,32,33]$. Therefore, in the teaching and learning of multiple varieties of a language, competence in an earlier variety serves as a prototype, against which a new variety is compared, processed, and then internalised as a system of variants.

Therefore, successful acquisition of one variety of a language, i.e., mastery of the prototype, can greatly facilitate acquisition of other varieties as systems of variants. To foreign language learners, it should be more advisable to consistently concentrate on learning one variety of a language until a prototype is successfully acquired [34]. Having multiple varieties combined as major sources of input may result in considerable confusion to learners, similar to confusion in balanced bilingualism, thus hampering internalisation of the prototypical structure. Although confusion due to concurrence of multiple languages can cease to exist in due course [28], there seems to be no evidence that confusion due to concurrence of multiple varieties will disappear in the same way.

\section{Interface Development in Learning Multiple Varieties}

Psychologically, how syntax-semantics (i.e., form-meaning) interfaces are developed is a core issue to understand second or additional language acquisition [35]. Interfaces can be understood as points of connection where different linguistic properties of a language interrelate with each other [36]. For instance, the connection between a word form and its meaning makes a form-meaning interface. As a form of additional language acquisition, the learning of a new language variety also hinges on successful interface development. However, how interfaces develop in the learning of a new language variety is strikingly under-explored. Theoretically, there should be at least four patterns of interface development in learning a new language variety, depending on whether new meaning or new form is processed and how they attach to or construct interfaces in the new variety.

When the learner of a new language variety encounters a new concept, one that is previously unknown to the learner but denoted by a lexical item in the new variety, the formmeaning interface will be developed in similar ways to learning an L1 [37]. The conceptual meaning must be comprehended in the first place, which is followed by attachment of its form to the meaning. We call this pattern of interface development conceptual evolution. For example, when a learner who has previously learnt French (as is spoken in France) learns words in Quebec French such as poutine (a dish of French fries and cheese curds topped with a brown gravy) and ouananiche (a small landlocked salmon of Lake St. John, Canada, and neighbouring waters), they must learn them as new concepts, which do not exist in French spoken in France. Nevertheless, when the concept denoted already exists in the learner's brain, attachment of the new form to the existing meaning will become the sole cognitive operation needed for interface development. Such development, instead of producing completely new interfaces, may only make more complex existing interfaces developed in the previously learnt variety or varieties, as a meaning is attached to by multiple forms. Such development is thus characterised by structural complicatedness of existing interfaces of the previously learnt variety or varieties, which can constitute another pattern of interface development, i.e., structural multiplication.

The third pattern of interface development can be observed in some other cases, which are probably rare. In those cases, meanings are neither added to the learner's brain as new ones, nor added with new forms from other varieties. Meanings are transferred from the form in one language variety to the form in another, which we call, also broadly researched as, conceptual transfer. Conceptual transfer refers to the cross-linguistic influence on concep- 
tual meaning [38]. For multilingual individuals, cross-linguistic effects are not confined to purely linguistic phenomena [39]. They go beyond phonological, syntactic, and semantic categories, and extend to how realities such as objects and actions are conceptualised, which is manifested in multilingual individuals' unique use of their linguistic repertoires for meaning conveyance [40].

A great number of studies have demonstrated that individuals who speak two or more languages possess a language transfer mechanism that can enable bidirectional transfer of conceptual meaning [39]. Such transfer of conceptual meaning from any one language to another is reflected in various lexical categories such as nouns, verbs, adjectives, etc. [40]. This indicates that multilingual individuals may have a complex conceptual system that consists of both sub-systems pertaining to specific languages and acquired connections between these sub-systems. As conceptual meaning is a direct result of interactions between the culture of a specific language and thinking of a particular individual or culture group [41], transfer of conceptual meaning in multilingual individuals reflects the interdependence between multiple languages, cultures, and thinking in multilingual contexts. Furthermore, the conceptual system of a multilingual individual, as is shaped by concepts from more than one language and culture, may essentially differ from that of a monolingual individual.

Individuals who speak multiple varieties of a language should likewise possess such a conceptual transfer mechanism. However, this mechanism may trigger conceptual transfers between varieties of a language less intensively and comprehensively than it probably can in individuals who speak multiple languages. Whilst different varieties of a language, like different languages, represent different cultures that are diversified in conceptual meaning, they share vast structural similarities. Such structural similarities can make conceptual meaning less transferable across different varieties of a language. On one hand, when structures of different language varieties are similar, they are more likely to be attached to the same meaning, which is a process of structural multiplication as is discussed above. On the other hand, when similar structures of different varieties mean different things, they can be processed as one form (showing variants though) with different meanings, which we call conceptual involution. This is the fourth pattern of interface development by which more meanings are involved within the same form. For instance, bienvenue only means "welcome" in French spoken in France, while in Quebec French it is also used to respond to others saying "thank you." In learning a new French variety, the learner has to add a new meaning to the old form, i.e., attaching an additional concept to the existing structure.

As can be seen, in either situation, i.e., structural multiplication or conceptual involution, conceptual transfer is unlikely to take place. Conceptual transfer across language varieties may occur on one crucial condition: the form-meaning interface in a previously learnt variety must "loosen" to the extent that the meaning can be detached and then reattached to the form in a new variety, or that the form in the new variety can be attached to the meaning which stands as a detached free body. That is to say, the meaning should be released from the interface of a previously learnt variety and nestle on the interface of the new variety.

Thus far, research dedicated to conceptual differences between varieties of a language has seemed to be scarce, although there is no shortage of studies that analyse and reveal structural differences between varieties of a language $[16,17,32,33]$. This paucity of research on conceptual differences between varieties of a language, and, subsequently, of research on transferability of conceptual meaning across different varieties, may have led to some misunderstanding of teaching and learning of multiple varieties as purely attaching, in learners' brains, extra meaning entries (derived from a later language variety) to existing lexical forms (belonging to an earlier language variety), or vice versa. Such misunderstanding has oversimplified conceptual transfer across varieties of a language as ordinary lexical learning, i.e., a process of enhancing structural-semantic well-formedness [39]. Therefore, it is imperative that we should reconsider the role of conceptual transfer in the teaching 
and learning of multiple varieties of a language, as opposed to conceptual transfer in that of multiple languages.

As elaborated above, the four patterns of interface development in the learning of multiple varieties, i.e., structural multiplication, conceptual involution, conceptual evolution, and conceptual transfer, are all underpinned by assemblage or re-assemblage of meanings and forms across language varieties. In teaching and learning multiple varieties, conceptual involution may play a more significant role in promoting receptive skills such as listening and reading. As the learner hears or reads a form that has multiple meanings, they need to make a choice about which variety the meaning should be selected from. In other words, the learner needs not only knowledge of the multiple meanings derived from multiple varieties, but also knowledge of correspondence between the lexical item and its variety. Such knowledge of correspondence can be conceptualised as variety markedness. Likewise, successful structural multiplication, which should affect productive skills more profoundly, is also characterised by variety markedness. When a learner tries to speak or write to communicate a meaning with multiple choices of form, they need to select the corresponding form in a particular language variety to enhance comprehensibility and appropriateness in a given situation. It should also be noted that in the discussion above, we have only elaborated on the development of internal interface, which is the syntax-semantics interface [42]; the development of external interface, i.e., syntax-discourse interface [43], may substantially differ from that of internal interface, thus requiring further research.

\section{Selected Language Practices of Multiple Varieties}

Socially, the teaching and learning of multiple varieties of a language may also differ from that of multiple languages, mainly in terms of an individual's personal language/variety use. Individuals' personal use of multiple languages can be brilliantly represented using the Dominant Language Constellations (DLC) framework [44]. A multilingual individual's DLC is a group of their "most important, vehicle languages as a whole," which "enabl[e] an individual to meet all needs in a multilingual environment." [45] (p. 19)

A multilingual individual's DLC reflects their selected language practices as they choose to use different languages in different social contexts [46] (p. 3). There should be at least two prerequisites for such selection, which previous studies do not seem to have probed into or elaborated upon. First, the multilingual individual must possess a repertoire that is large enough in all languages to be used. In the repertoire, all languages are not necessarily equal in terms of linguistic competence, but each should provide the individual with sufficient proficiency for use in a given context. Such a repertoire constitutes the linguistic prerequisite for selected language practices. Second, selection of any or all of the languages for use is closely associated with a multilingual individual's agency as an agentive prerequisite. In other words, only when the individual has willingness that is strong enough (or resistance which is low enough) to use one or more languages in a communication situation can selected language practices be enacted [47]. Before a multilingual individual actually conducts selected language practices to engage in daily interactions, to position themselves and be positioned by others [48], or to develop their trajectory of multiple language use [44], they first need a proper linguistic repertoire and the propelling willingness to use this repertoire.

These two prerequisites seem to have been neglected by researchers who have taken the DLC approach to primarily investigate the learning and use of multiple languages rather than of multiple varieties. This may be partly because multilingual individuals who use multiple languages should, by default, possess required linguistic competences and willingness to communicate [47]. However, multilingual individuals who use multiple language varieties may not, or do not even have to, possess such competences and willingness, as Shan and $\mathrm{Xu}$ [49] revealed in a study that examines the use of South African English (a variety which was not previously learnt) by three Chinese learners of English as a foreign language who worked in South Africa as journalists. Since their arrival in South Africa, the three English learners had been exposed to South African English and had grad- 
ually been able to understand local people's conversations. Although they acquired fairly practical receptive skills, they did not seem to advance greatly in productive skills. In our interviews, they reported that they were reluctant to learn to speak South African English, but that they were still able to make themselves understood in work settings with their British or American English varieties. As can clearly be seen in this example, multilingual individuals' linguistic competence in a particular variety may not be compatible with all language needs for that variety, because their competence in another variety can be utilised to compensate, at least to a certain extent, for the deficiency.

As regards willingness to use a language variety, multilingual individuals' selected language practices concerning a particular variety may be affected by their language attitudes towards that variety [50] and also by the ideal selves they envisage in their future life [51]. Shan and $\mathrm{Xu}$ [49] have also found a Chinese learner of French (as is spoken in France) who worked in Brussels uninterested in learning or picking up Belgian French, because he did not have a strong desire for integrating into the mainstream community. To sum up, multilingual individuals of multiple language varieties may not share with those of multiple languages the linguistic and agentive prerequisites for selected language practices, which obviously warrants systematic further investigation. For a multiple variety speaker, although it may also hamper communication, the absence of the two prerequisites can be adequately compensated for by the speaker's repertoire of a substitute variety.

\section{Conclusions}

Thus far, we have analysed, through comparison with multiple languages, the linguistic, psychological, and social characteristics of multiple language varieties as taught and learnt to promote multilingual literacies, thus promoting sustainable personal development through multilingual education. Linguistically, acquisition of language varieties is a process of assimilating variants from a new variety into an earlier variety, which serves as a prototype system. Such assimilation is a psychological project of form-meaning interface development, which may follow the patterns of structural multiplication, conceptual involution, conceptual evolution, or/and conceptual transfer. When multiple language varieties are actually used in social contexts, multilingual individuals' selected language practices may be supported by their combined linguistic resources from multiple varieties rather than depend on a single variety despite its dominance in a given situation.

Although the analysis presented in this article is theoretical, and, to some extent, hypothetical, it still carries some pedagogical implications for sustainable multilingual education, particularly for the teaching and learning of foreign languages such as English, French, Spanish, Arabic, etc., which have multiple varieties. By and large, the teaching and learning of multiple language varieties should be more usage-based rather than ruleoriented and we should pay more attention to both the diversity and special properties of interface development in multiple variety learning. Moreover, the DLC approach, when applied to multiple language varieties, may need proper modifications so as to better inform selected language practices involving multiple varieties in a multilingual context.

Author Contributions: Conceptualisation of Section 3, H.X.; conceptualisation of Sections 2 and 4, H.X. and Z.S.; writing — original draft preparation, H.X.; writing—review and editing, Z.S.; funding acquisition, Z.S. Both authors have read and agreed to the published version of the manuscript.

Funding: This research was funded by the Fundamental Research Funds for the Central Universities in Beijing Foreign Studies University, grant number 2021JJ012.

Institutional Review Board Statement: Not applicable.

Informed Consent Statement: Not applicable.

Data Availability Statement: Not applicable.

Acknowledgments: We sincerely thank the anonymous referees and the editors for their constructive and valuable comments and suggestions, which have helped us tremendously improve the manuscript. 
Conflicts of Interest: The authors declare no conflict of interest.

\section{References}

1. Hornberger, N.H.; McKay, S.L. Sociolinguistics and Language Education; Multilingual Matters: Bristol, UK, 2010.

2. Cenoz, J.; Hufeisen, B.; Jessner, U. The Multilingual Lexicon; Springer: New York, NY, USA, 2003.

3. Lau, S.M.C.; Van Viegen, S. Plurilingual Pedagogies; Springer: Berlin/Heidelberg, Germany, 2020.

4. Weinreich, U. Language in Contact: Findings and Problems; Mouton Publishers: The Hague, The Netherlands, 1953.

5. Wallace, C. Literacy and the Bilingual Learner; Springer: Berlin/Heidelberg, Germany, 2013.

6. Harris, P.; Brock, C.; McInnes, E.; Neill, B.; Diamond, A.; Carter, J.; Camaitoga, U.; Krishna, M.; Giannakis, E. Children's Multilingual Literacy. In International Perspectives on Early Childhood Education and Development; Springer: Berlin/Heidelberg, Germany, 2020.

7. Cockcroft, K.; Wigdorowitz, M.; Liversage, L. A multilingual advantage in the components of working memory. Biling. Lang. Cogn. 2019, 22, 15-29. [CrossRef]

8. Greve, W.; Koch, M.; Rasche, V.; Kersten, K. Extending the scope of the 'cognitive advantage' hypothesis: Multilingual individuals show higher flexibility of goal adjustment. J. Multiling. Multicult. Dev. 2021, 42, 1-17. [CrossRef]

9. Festman, J.; Schwieter, J.W. Self-concepts in reading and spelling among mono- and multilingual children: Extending the bilingual advantage. Behav. Sci. 2019, 9, 39. [CrossRef] [PubMed]

10. Spellerberg, S.M. Metalinguistic awareness and academic achievement in a linguistically diverse school setting: A study of lower secondary pupils in Denmark. Int. J. Multiling. 2015, 13, 19-39. [CrossRef]

11. Sörman, D.E.; Josefsson, M.; Marsh, J.E.; Hansson, P.; Ljungberg, J.K. Longitudinal effects of bilingualism on dual-tasking. PLoS ONE 2017, 12, e0189299. [CrossRef]

12. Lüdi, G.; Py, B. To be or not to be-A plurilingual speaker. Int. J. Multiling. 2009, 6, 154-167. [CrossRef]

13. Marshall, S.; Moore, D. 2B or not 2B plurilingual? Navigating languages literacies, and plurilingual competence in postsecondary education in canada. TESOL Q. 2013, 47, 472-499. [CrossRef]

14. Gao, X.; Zheng, Y. Multilingualism and higher education in Greater China. J. Multiling. Multicult. Dev. 2019, 40, 555-561. [CrossRef]

15. Baratta, A. World Englishes in English Language Teaching; Springer: Berlin, Germany, 2019.

16. Reutner, U. Manuel des Francophnies; De Gruyter: Berlin, Germany, 2017.

17. Robillard, D.D.; Beniamino, M. Le Français dans L'espace Francophone: Description Linguistique et Sociolinguistique de la Francophonie; Champion: Paris, France, 1993; Volume 1.

18. Chantal, L. Standard French: Synthetic elements. In Varieties of French Spoken in French-Speaking Space: Resources for Teaching; Detey, S., Durand, J., Laks, B., Lyche, C., Eds.; Ophrys: Paris, France, 2010; pp. 143-165.

19. Leitner, G. English as a pluricentric language. In Pluricentric Languages; Clyne, M., Ed.; De Gruyter: Berlin, Germany, 1991; pp. 179-238.

20. Saville-Troike, M. Introducing Second Language Acquisition, 2nd ed.; Cambrige University Press: Cambridge, UK, 2012.

21. Saraceni, M. World Englishes: A Critical Analysis; Bloomsbury: London, UK, 2015.

22. Yang, C. Three factors in language variation. Lingua 2010, 120, 1160-1177. [CrossRef]

23. Falk, Y.; Bardel, C. Object pronouns in German L3 syntax: Evidence for the L2 status factor. Second Lang. Res. 2010, $27,59-82$. [CrossRef]

24. Oschwald, J.; Schättin, A.; Von Bastian, C.C.; Souza, A.S. Bidialectalism and bilingualism: Exploring the role of language similarity as a link between linguistic ability and executive control. Front. Psychol. 2018, 9, 1997. [CrossRef]

25. Studdert-Kennedy, M.; Terrace, H. In the beginning: A review of Robert C. Berwick and Noam Chomsky's why only us. J. Lang. Evol. 2017, 2, 114-125. [CrossRef]

26. Lehmann, C. Linguistic competence: Theory and empiry. Folia Linguist. 2007, 41, 223-278. [CrossRef]

27. García, A.M.; Muñoz, E.; Kogan, B. Taxing the bilingual mind: Effects of simultaneous interpreting experience on verbal and executive mechanisms. Biling. Lang. Cogn. 2019, 23, 729-739. [CrossRef]

28. Weber, R.C.; Johnson, A.Y.; Riccio, C.A.; Liew, J. Balanced bilingualism and executive functioning in children. Biling. Lang. Cogn. 2015, 19, 425-431. [CrossRef]

29. Sharma, D. Typological diversity in New Englishes. Engl. World Wide 2009, 30, 170-195. [CrossRef]

30. Von Stutterheim, C.; Bouhaous, A.; Carroll, M. From time to space: The impact of aspectual categories on the construal of motion events: The case of Tunisian Arabic and modern standard Arabic. Linguistics 2017, 55, 207-249. [CrossRef]

31. Van Der Slik, F.; Van Hout, R.; Schepens, J. The role of morphological complexity in predicting the learnability of an additional language: The case of La (additional language) Dutch. Second Lang. Res. 2017, 35, 47-70. [CrossRef]

32. Trudgill, P.; Hannah, J. International English, 6th ed.; Routledge: London, UK, 2017.

33. Detey, S.; Durand, J.; Laks, B.; Lyche, C. Varieties of Spoken French; Oxford University Press: Oxford, UK, 2016.

34. $\mathrm{Xu}, \mathrm{H}$. Applying "variety consistency" and "communicative responsiveness" in teaching English as an international language in China: A conceptual framework. J. Lang. Policy Lang. Plan. 2021, 8, 1-10.

35. Plonsky, L.; Marsden, E.; Crowther, D.; Gass, S.M.; Spinner, P. A methodological synthesis and meta-analysis of judgment tasks in second language research. Second Lang. Res. 2019, 36, 583-621. [CrossRef] 
36. Montrul, S. Multiple interfaces and incomplete acquisition. Lingua 2011, 121, 591-604. [CrossRef]

37. Pexman, P. The role of embodiment in conceptual development. Lang. Cogn. Neurosci. 2017, 34, 1274-1283. [CrossRef]

38. Adamou, E.; De Pascale, S.; García-Márkina, Y.; Padure, C. Do bilinguals generalize estar more than monolinguals and what is the role of conceptual transfer? Int. J. Biling. 2018, 23, 1549-1580. [CrossRef]

39. Jarvis, S. Conceptual transfer: Crosslinguistic effects in categorization and construal. Biling. Lang. Cogn. 2010, 14, 1-8. [CrossRef]

40. Wolter, B.; Yamashita, J.; Leung, C.Y. Conceptual transfer and lexical development in adjectives of space: Evidence from judgments, reaction times, and eye tracking. Appl. Psycholinguist. 2020, 41, 595-625. [CrossRef]

41. Ji, L.-J.; Zhang, Z.; Nisbett, R.E. Is it culture or is it language? Examination of language effects in cross-cultural research on categorization. J. Pers. Soc. Psychol. 2004, 87, 57-65. [CrossRef] [PubMed]

42. Tsimpli, I.M.; Sorace, A. Differentiating interfaces: L2 performance in syntax-semantics and syntax-discourse phenomena. In Proceedings of the BUCLD 30: Proceedings of the 30th Annual Boston University Conference on Language Development, Boston, MA, USA, 46 November 2005; Bamman, D., Magnitskaia, T., Zaller, C., Eds.; Cascadilla Press: Somerville, MA, USA, 2006; Volume 2, pp. 653-664.

43. Sorace, A.; Serratrice, L. Internal and external interfaces in bilingual language development: Beyond structural overlap. Int. J. Biling. 2009, 13, 195-210. [CrossRef]

44. Lo Bianco, J.; Aronin, L. Dominant Language Constellations; Springer: Berlin/Heidelberg, Germany, 2020.

45. Aronin, L. Dominant language constellations as an approach for studying multilingual practices. In Explorations into Chinese as a Second Language; Springer: Berlin/Heidelberg, Germany, 2020; pp. 19-33.

46. Bianco, J.L.; Aronin, L. Introduction: The dominant language constellations: A new perspective on multilingualism. In Explorations into Chinese as a Second Language; Springer: Berlin/Heidelberg, Germany, 2020; pp. 1-15.

47. Peng, J.-E. Understanding willingness to communicate as embedded in classroom multimodal affordances: Evidence from interdisciplinary perspectives. Linguist. Educ. 2019, 51, 59-68. [CrossRef]

48. Gu, M.; Patkin, J.; Kirkpatrick, A. The dynamic identity construction in English as lingua franca intercultural communication: A positioning perspective. System 2014, 46, 131-142. [CrossRef]

49. Shan, Z.; Xu, H. Dominant "variety" constellations? Selected language practices by foreign language learners of Englishes and Frenches. Int. J. Multiling. 2021. in progress.

50. Si, J. English as a native language, world Englishes and English as a lingua franca-informed materials: Acceptance, perceptions and attitudes of Chinese English learners. Asian Engl. 2019, 21, 190-206. [CrossRef]

51. Henry, A.; Thorsen, C. The ideal multilingual self: Validity, influences on motivation, and role in a multilingual education. Int. J. Multiling. 2017, 15, 349-364. [CrossRef] 